\title{
Políticas públicas y participación ciudadana: análisis de tres programas estatales implementados en un barrio popular de Mar del Plata (2018-2020)
}

Ispizúa, Jeremías

Becario de investigación del CONICET. Especialista en Diseño estratégico de tecnologías para el desarrollo inclusivo sustentable. Facultad de Arquitectura, Urbanismo y Diseño. Universidad Nacional de Mar del Plata.

jereispizua@gmail.com

\section{Melián, Isaac}

Becario de investigación del CONICET. Especialista en Diseño estratégico de tecnologías para el desarrollo inclusivo sustentable. Facultad de Arquitectura, Urbanismo y Diseño. Universidad Nacional de Mar del Plata.

jose.isaac.melian@gmail.com

Mitidieri, Antonela

Becaria de investigación UNMdP. Facultad de Arquitectura, Urbanismo y Diseño. Universidad Nacional de Mar del Plata. Magister en Planejamento e Gestão do Territorio (UFABC). antomitidieri4@gmail.com

\section{Blanco Pepi, Macarena}

Becaria de investigación del CONICET. Facultad de Arquitectura, Urbanismo y Diseño. Universidad Nacional de Mar del Plata.

macablancopepi@gmail.com

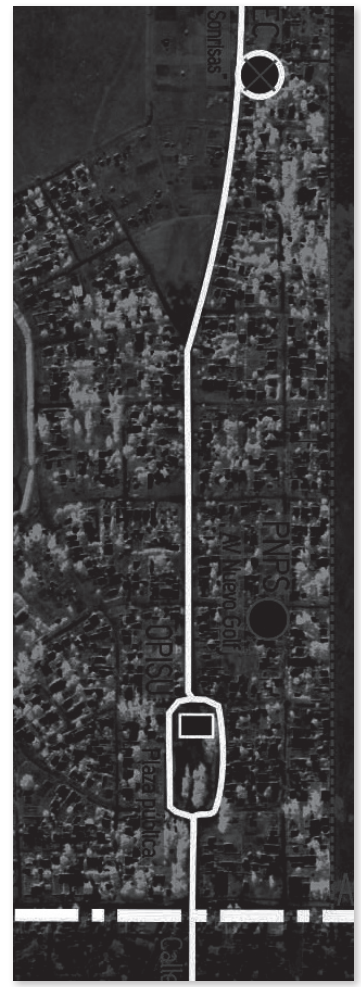

http://dx.doi.org/10.30972/crn.31315779 


\section{Políticas públicas y participación ciudadana: análisis de tres programas estatales implementados en un barrio popular de Mar del Plata (2018-2020)}

\section{Resumen}

En este trabajo se presentan los resultados del análisis de tres programas estatales implementados en el asentamiento de mayor dimensión de la ciudad de Mar del Plata. Se plantea en el contexto de una investigación más amplia en tecnologías sociales y hábitat popular en territorios de alta exclusión, donde el grupo de investigación participa activamente de los procesos que estudia. Se vinculan tres ejes: el análisis de políticas públicas, la gestión alternativa y la urbanización popular, con el objetivo de indagar en las diversas formas de gestión de cada experiencia. Para eso se caracterizan los distintos actores que participan, sus interacciones y los modos de vinculación con la sociedad civil. Reflexionar en ese sentido contribuye a pensar políticas de integración socio-urbana en contextos de pobreza con mayor anclaje en territorialidades locales. Las técnicas y métodos empleados para el trabajo en el territorio se basan en la identificación de situaciones problemáticas y alianzas entre actores sociales para co-construir estrategias problema-solución.

\section{Palabras clave}

Hábitat popular; políticas públicas; co-gestión; participación ciudadana.
Public policies and citizen participation. Analysis of three state programs implemented in a popular neighborhood of Mar del Plata (2018-2020)

\begin{abstract}
This paper presents the results of the analysis of three state programs implemented in a sprawling poor neighborhood in Mar del Plata city. It arises in the context of broader research on social technologies and popular habitat in highly excluded territories, where the research group actively participates in the processes it studies. Three axes are linked: the analysis of public policies, alternative management and popular urbanization with the aim of investigating the different forms of management of each experience. For this purpose, the different actors involved, their interactions and the ways of linking with civil society are characterized. Reflecting on this issue contributes to thinking about socio-urban integration policies in contexts of poverty with greater anchorage in local territorialities. The techniques and methods used for work in the territory are based on the identification of problematic situations and alliances between social actors to co-construct problem-solution strategies.
\end{abstract}

\section{Keywords}

Popular habitat; public policies; co-management; citizen participation.

\section{Políticas públicas e participação cidadã: análise de três programas estaduais implementados num bairro popular de Mar del Plata (2018-2020)}

\section{Resumo}

Este artigo apresenta os resultados da análise de três programas estaduais implantados no maior assentamento da cidade de Mar del Plata. Surge no contexto de uma pesquisa mais ampla sobre tecnologias sociais e habitats populares em territórios altamente excluídos, onde o grupo de pesquisa participa ativamente dos processos que estuda. Três eixos estão interligados: a análise das políticas públicas, a gestão alternativa e a urbanização popular com o objetivo de investigar as diversas formas de gestão de cada experiência. Para isso, são caracterizados os diferentes atores que participam, suas interações e as formas de vinculação com a sociedade civil. Refletir neste sentido contribui para pensar as políticas de integração sócio urbana em contextos de pobreza com maior ancoragem nas territorialidades locais. As técnicas e métodos utilizados para o trabalho no território baseiam-se na identificação de situações problemáticas e alianças entre atores sociais para co-construir estratégias de solução de problemas.

\section{Palavras chave}

Habitat popular; políticas públicas; co-gestão; participação cidadã. 


\section{Planteo del problema y diálogo con autores de referencia}

Desde la década del 70, los modos de intervención del Estado en Argentina, caracterizados por desregulaciones y privatizaciones, dieron lugar a procesos emergentes en múltiples dimensiones en términos territoriales y sociales. La agudización de la exclusión social ha estimulado desde entonces procesos de urbanización popular, en los cuales el acceso a la tierra, la vivienda y la infraestructura de servicios se produce mediante esfuerzos de autogestión de recursos que incluyen prácticas y estrategias colectivas.

En la urbanización popular, las irregularidades ${ }^{1}$ son la norma para el acceso a la tierra, la producción de viviendas y servicios, los cuales son administrados o construidos parcialmente fuera de la lógica del mercado y sin el apoyo del Estado (Pirez, 2015; Cravino Et AL., 2010). Cada territorio, entendido como un campo de tensiones y disputas de múltiples actores que precisan ser identificados, reconocidos y problematizados (GARGANTINI ET AL., 2018), exige un tipo de gestión para satisfacer necesidades específicas. Sin embargo, es común encontrarse con soluciones estandarizadas y estereotipadas ofrecidas desde el Estado e implementadas sin tener en cuenta las demandas locales.

Interesa en este punto la distinción que hace PIrez (1995) sobre la gestión de la ciudad, en la cual caracteriza a los actores que producen ciudad desde distintas lógicas: así se realizan procesos orientados predominantemente por la obtención de ganancia (empresarios individuales, empresas, conglomerados de empresas que realizan actividades dentro del mercado); orientados por la lógica de la necesidad, a través de acciones colectivas o individuales, de organización comunitaria de los sectores populares; por la lógica del conocimiento, que contribuyen a las decisiones de los anteriores y, en tal sentido, introducen perspectivas que forman parte de esas decisiones y, por último, desde una lógica política, los actores gubernamentales. Esta "producción de la ciudad" se da en un sentido determinado según cómo sean sus articulaciones y la alineación de los actores.

Comprender las tensiones y contradicciones, pero también las alianzas y vinculaciones que se producen para acceder a derechos humanos básicos implica considerar la dimensión territorial, política y conflictual de la urbanización popular (CACOPARDO ET AL., 2018). Abordar el problema social desde el territorio significa considerar y reconocer otras formas de
1. Regular-irregular, formalinformal, legal-ilegal son categorías que implican un cuestionamiento sobre cómo es definida esa denominación, ya que está directamente asociada a normas y leyes creadas al servicio de un sistema que pretende definir criterios según los valores de legalidad que históricamente favorecen a una hegemonía dominante. 
interactuar con el Estado, a partir de circuitos socio-territoriales, en que las problemáticas sean determinadas principalmente por la sociedad civil y las estructuras de gobierno acompañen y faciliten en sus espacios políticos más flexibles.

La gestión alternativa que acompaña estos procesos conflictivos y heterogéneos parte del entendimiento del rol del Estado no como el único actor capaz de implementar estrategias de acción, sino como uno de los tantos actores que conforman redes de co-gestión. Las redes de gestión inter-actorales y multisectoriales son factibles de ser implementadas si se tiene en cuenta una nueva concepción del Estado como redistribuidor del poder con criterios de equidad (Peli, 2007). De esta forma, confronta los modelos de gestión dominantes y propone pautas de transformación con perspectiva interactoral, multisectorial y participativa para el abordaje de los problemas planteados.

En esta línea se identifican investigaciones que se realizan a partir de prácticas territoriales, en las cuales se propone aportar soluciones a partir de estrategias de gestión interinstitucional y de desarrollo local con fuerte anclaje territorial, para trabajar en forma participativa con problemas socio-habitacionales (CAcopardo et Al., 2018; Peyloubet, 2012; Pelli, 2007). Estas estrategias se traducen en modelos de gestión alternativos, que efectivizan posibilidades de recursos municipales, provinciales y nacionales y propician la promoción y gestión intersectorial de grupos sociales relevantes a fin de promover prácticas emancipadoras.

En este trabajo se realiza un análisis de tres planes y programas estatales que se materializaron a través de la interacción con la sociedad civil, implementados en el asentamiento de mayor dimensión de la ciudad de Mar del Plata en el período 2018-2019. Se plantea en el contexto de una investigación más amplia en tecnologías sociales y hábitat popular en territorios de alta exclusión, donde el grupo de investigación participa activamente de los procesos que estudia.

El objetivo es indagar en las diversas formas de gestión de cada experiencia a partir de caracterizar a los distintos actores que participan, sus interacciones y los modos de vinculación con la sociedad civil. En este sentido, interesa contribuir con materiales e insumos de reflexión a la siguiente pregunta: ¿cómo pensar políticas de integración socio-urbana en contextos de pobreza con mayor anclaje en territorialidades locales? 


\section{Abordaje analítico-metodológico}

Las técnicas y métodos empleados para el trabajo en el territorio se basan en la identificación de situaciones problemáticas y en alianzas entre actores sociales para co-construir estrategias problema-solución. Dentro de la estructura de gestión, de la cual el grupo de investigación forma parte, las vecinas, vecinos y sus redes son el centro de las vinculaciones, a la vez que son generadores de estrategias para la solución de problemas y desarrollos.

El recorte temporal abarca el inicio de cada política pública en el barrio y se extiende hasta noviembre de 2019. La participación directa en los procesos que se estudian permite varios registros:

1. Del período anterior a la implementación de las políticas (trayectoria territorial).

2. De las discusiones de implementación de las políticas públicas (debido a la participación en mesas de trabajo, diseño y selección de equipos de gestión y planificación de algunas de sus actividades).

3. De los flujos de información sobre las dificultades de ejecución de los programas (obtenidos de información verbal y de entrevistas semiestructuradas).

4. De discursos y testimonios en la propaganda y comunicación de las políticas públicas (hecho que devela el posicionamiento ideológico de los decisores políticos).

Para este artículo se adopta una perspectiva que aborda la política pública desde el ángulo específico de su "lógica de acción", siendo el punto de partida la arena en la que los actores político-administrativos y sociales interactúan en un tiempo y un espacio determinados (SUBIRATS ET AL., 2008). En el plano de interacción Estado-sociedad existen acciones que pueden o no ser consideradas políticas públicas. En este artículo, el término política pública abarca desde los planes y programas hasta los discursos y tomas de posición de los representantes cuando hablan en nombre del Estado ante una cuestión socialmente problematizada (Oszlak \& O’Donnell, 1995). Si bien se verán algunas cuestiones relacionadas con el diseño de las políticas, es importante señalar que de aquí en adelante el foco estará sobre lo que OszLak llama plano funcional, es decir, el escenario donde tiene lugar la participación de la sociedad civil en sus diferentes formas. Las vinculaciones interactorales expuestas ocurren en la ejecución ${ }^{2}$ de políticas públicas, y aquí no solo interesa determinar las modalidades de participación, sino también de qué forma los planes y pro-
2. Según OszLaK la participación ciudadana se puede dar en tres tiempos: pasado, presente y futuro, es decir, en el diseño y planificación, en la ejecución o en la evaluación y control de los resultados a partir de la experiencia. 
gramas se adaptan a la presencia de redes preexistentes. Para ello se plantea analizar los programas a través de cuatro dimensiones que serán atravesadas por las articulaciones generadas en cada una.

\section{Trayectoria socio-técnica}

Se utiliza este concepto para analizar de qué forma los actores presentes en el territorio construyen las relaciones problema-solución alrededor de una cuestión antes de la ejecución de una política pública (THomas eT AL., 2012). El estudio de este período previo enriquece el saber acerca del poder relativo de los actores, la naturaleza de sus recursos, percepciones e ideologías, alianzas y conflictos y las estrategias de acción política.

\section{Modalidad de participación ciudadana}

Esta dimensión permite identificar el tipo y grado de participación que tiene la sociedad civil en la implementación de las políticas públicas puestas en cuestión. Incluye dos variables de análisis:

- Rol del referente territorial: entendido como aquel miembro de la sociedad, con un rol de promotor o líder (OszLAK, 2009), con conocimiento experto no científico que regenera en su hacer, pensar y decir una nueva versión mucho más cercana de la realidad considerando el recorte de su propia experiencia en el territorio (PeyLoubet, 2017).

- Formas jurídicas de quienes participan en estos procesos, a efectos de entender el significado y la valoración que tienen en la vinculación Estado-sociedad los distintos tipos de intermediarios. OszLAK habla de ellos como el tercer y el cuarto sector, haciendo referencia a las formalidades que cumple una organización de la sociedad civil constituida como tal, sus capacidades y entendimientos respecto de organizaciones que enfrentan la resolución de problemas que afectan su vida cotidiana, sin contar con los recursos materiales y organizacionales necesarios para lograr una existencia relativamente permanente e institucionalizada.

\section{Momento de implementación y período de ejecución de la política}

Tomar en cuenta el momento histórico de surgimiento de cada política y el período de implementación son dos aspectos que ayudan a identificar el objetivo real de la intervención por parte del Estado, sobre todo cuando suceden alrededor de una problemática tan compleja y multidimensional como la integración social y urbana de las llamadas “villas” y "asentamientos". 


\section{Recursos materiales de una política pública}

Esta dimensión de análisis está destinada a indagar cómo se efectivizan y se materializan las herramientas y recursos que una política ofrece. Estos elementos se comprenden a partir de lo que las políticas manifiestan en su reglamentación, las comunicaciones de los funcionarios políticos informales y formales, lo que efectivamente sucede en la ejecución y las posibilidades reales de esos recursos.

\section{Presentación de casos de estudio y su análisis}

\subsection{Tres procesos de gestión de programas estatales en el barrio Nuevo Golf}

Mar del Plata, ciudad argentina de escala intermedia situada dentro de la provincia de Buenos Aires, con una población de 653.406 habitantes $^{3}$, es la segunda urbe turística más significativa del país, debido a su importante zona balnearia y portuaria. La ciudad crece según las características de las ciudades intermedias latinoamericanas, con su desigualdad condicionada por las fuerzas del mercado, los intereses del sector inmobiliario y las políticas exclusivas de un Estado que invisibiliza los sectores de mayor vulnerabilidad.

La ciudad y su partido, General Pueyrredon, han sido objeto de diversos relevamientos ${ }^{4}$ que demuestran serias dificultades para determinar el número real de familias que viven en condiciones precarias, así como su ubicación en la ciudad. Aunque no es el objeto de este artículo comprender las implicaciones políticas que pueden tener las mediciones y la caracterización de los asentamientos, es importante señalar que la difusión de datos imprecisos repercute en el diseño de herramientas que son la base para la construcción de las políticas públicas y la gestión urbana (MitidiERI ET AL., 2019).

La fuente de datos más cercana es la municipal, que en el año 2017 realizó un relevamiento según los parámetros establecidos por la Ley 14.449 de la Provincia de Buenos Aires sobre Acceso Justo al Hábitat (2013). El informe ${ }^{5}$ indica que hay un total de 114 villas y asentamientos (figura 1) precarios en el partido, con 10.300 familias que viven en estas condiciones, dentro de un total aproximado de 327 hectáreas.

En el barrio Nuevo Golf (figura 2) se emplaza el asentamiento "irregular/precario" de mayor dimensión y que además presenta un rápido crecimiento poblacional: en tan solo
3. Disponible en: http://www. estadistica.ec.gba.gov.ar/ dpe/index.php/poblacion/ proyecciones/municipios Acceso el 23 de octubre de 2019 .

4. En siete años se han llevado a cabo cinco relevamientos de villas y asentamientos en el Partido de General Pueyrredon: Fundación Techo (2013, 2016), Ministerio de Infraestructura y Servicios Públicos de la Provincia de Buenos Aires (2014), Dirección Social de Vivienda de la Municipalidad de General Pueyrredon (2017) y en el nivel nacional se creó el Registro Nacional de Barrios Populares (RENABAP, 20162017).

5. Informe sobre Villas y Asentamientos de Mar del Plata, Secretaría de Planeamiento Urbano, MGP (agosto de 2017). 


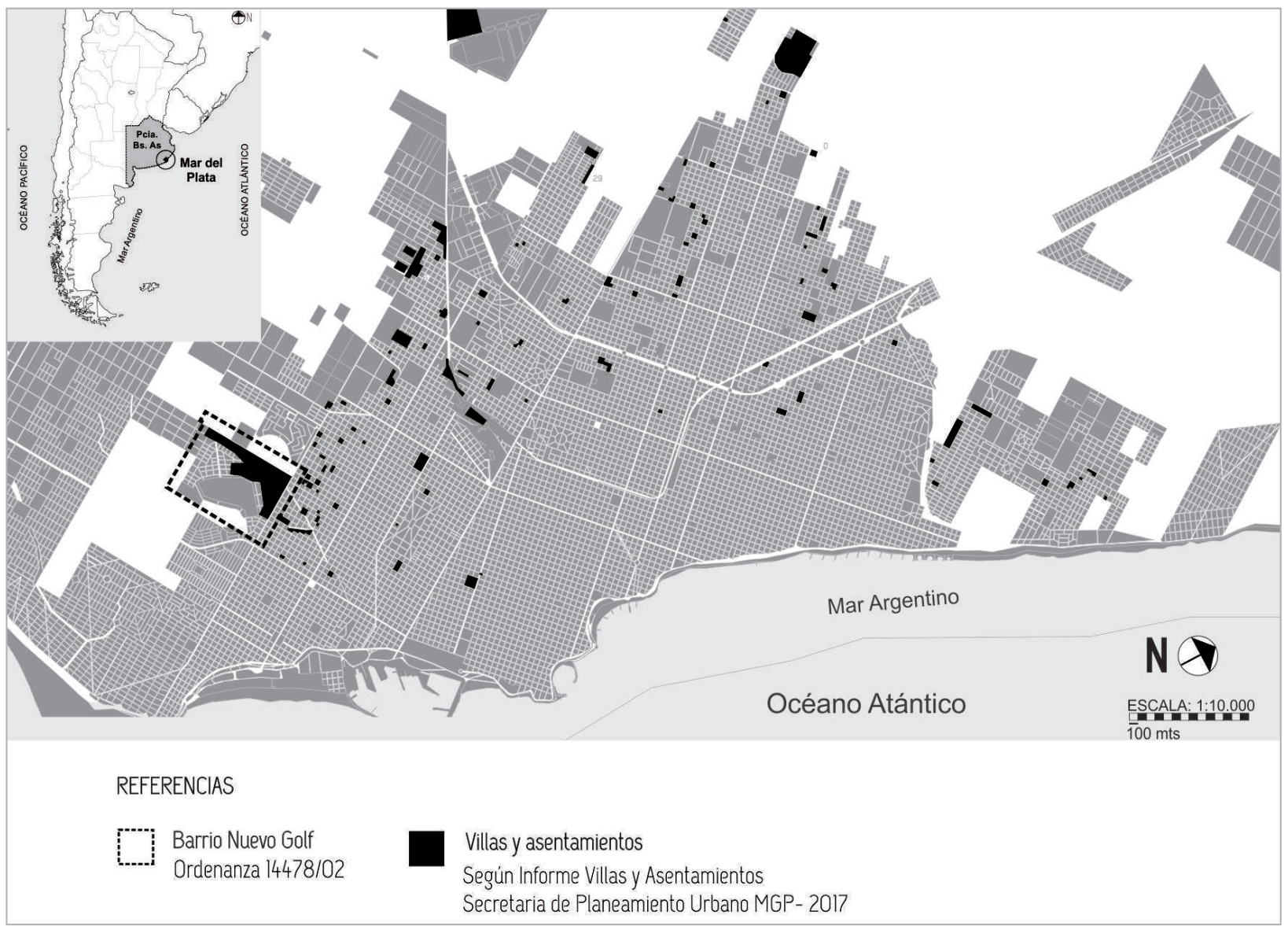

Figura 1. Villas y asentamientos de la ciudad de Mar del Plata y barrio Nuevo Golf dos años aumentó un tercio su población al incrementar el número de familias de 700 en 2015 a 1000 familias en 2017. Se trata de un barrio relativamente joven, surgido en el marco de la crisis de los años 2001-2002. 
Políticas públicas y participación ciudadana: análisis de tres programas estatales implementados en un barrio popular de Mar del Plata (2018-20२०)

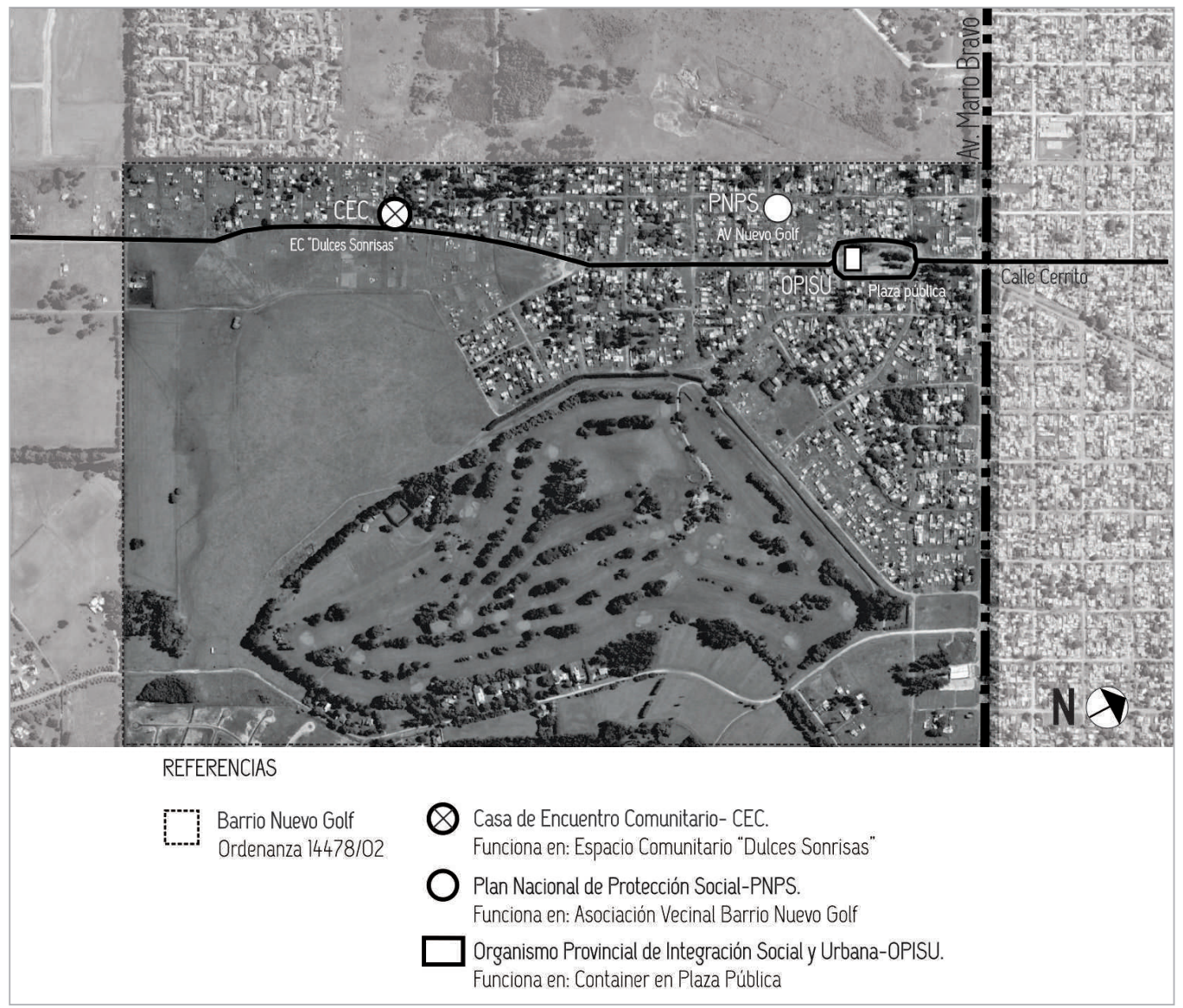

Figura 2. Geolocalización de espacios de referencia y programas estatales en barrio Nuevo Golf

La ausencia del Estado para dar solución a las problemáticas y necesidades ha motivado acciones vecinales y articulaciones de cooperación con diversas instituciones y organizaciones externas al territorio. Dos actores sociales relevantes que influyen en la agenda estatal 
6. Dentro de los procesos y conquistas más significativas, podemos mencionar la obra de mejoramiento en el acceso del barrio y el pavimento de la calle Cerrito, principal vía de circulación (MITIDIERI ET AL., 2019) sobre el barrio son Mario y Andrea. Mario preside la Asociación Vecinal (en adelante AV) y Andrea es referente del espacio "Comedor Dulces Sonrisas". Ambos vecinos ejercen roles significativos en las luchas para activar procesos de urbanización popular. Desde la AV, Mario ha contribuido en procesos referidos a la gestión urbana y al mejoramiento de la infraestructura local (transporte, saneamiento, accesibilidad, iluminación, salud). Desde el comedor, Andrea se ha ocupado de atender problemáticas de la alimentación en la niñez. En ambos espacios, el grupo de investigación, a través de su estructura de gestión, buscó efectivizar las redes existentes para acompañar los procesos de transformación social y urbanización popular emergentes de estos actores.

Recientemente, organismos estatales reconocieron la capacidad de gestión de los actores y decidieron implementar conjuntamente políticas de urbanización y desarrollo social y comunitario $^{6}$. Desde 2018 se pusieron en marcha tres políticas públicas: Casa de Encuentro Comunitario (CEC), Organismo Provincial de Integración Social y Urbana (OPISU) y Programa Nacional de Protección Social (PNPS) (figura 2). Las características de cada política pública y la forma en que se ejecutan en el territorio serán descritas a continuación, a partir de una lógica narrativa y diacrónica de los hechos.

\section{Casa de Encuentro Comunitario}

Para analizar la trayectoria del programa CEC es necesario remontarse a 2013, cuando el grupo de investigación comenzó a realizar acciones conjuntas en mejoramiento de viviendas a través de diferentes técnicas ligadas a autoconstrucción del hábitat popular. La referente Andrea, quien participaba de las actividades, comenzó a ofrecer una copa de leche a las niñas y los niños que acompañaban a sus padres y madres en las jornadas de autoconstrucción. Esta actividad sostenida semana a semana en su casilla continuó evolucionando, y a principios de 2015, como respuesta a la crisis alimentaria, la referente reforzó la ayuda ofreciendo también comida y complementando con otras actividades.

La estrategia de gestión del espacio se sustentaba sobre la base de donaciones de particulares y aportes propios. Un año más tarde, una ONG ligada a la promoción del hábitat se sumó a la labor solidaria de la referente y se conformó una red de gestión que se proponía la co-construcción de un nuevo espacio físico de uso comunitario. El desmantelamiento de la casilla donde todo comenzó se convirtió en un hito que marcó la conciencia colectiva de quienes hoy participan de la red. 
La trayectoria del grupo de investigación, con vínculo permanente y continuado con la referente y otros actores de la red, creó las condiciones de posibilidad para el desembarco de la agencia estatal y la formación de un equipo de gestión para la implementación del Programa "Casa de Encuentro Comunitario"7. El Programa CEC, perteneciente al Ministerio de Desarrollo Social de la Provincia de Buenos Aires, tiene como objetivo promover políticas de integración y participación comunitaria que tiendan al fortalecimiento de las Organizaciones de la Sociedad Civil (OSC) y promuevan el desarrollo familiar y comunitario. La ONG armó un equipo técnico conformado por una coordinadora, dos trabajadores sociales y personal administrativo sobre la base de una estructura organizacional, prediseñada por el Estado. Esta estructura ofrecía cierto grado de flexibilidad para que las partes ejecutoras diseñaran el equipo técnico sobre la base de las necesidades territoriales, autonomía considerada indispensable por la ONG.

El ministerio subsidió el programa a través de desembolsos mensuales administrados por la ONG. Este monto cubre gastos de mantenimiento del espacio físico y talleristas/docentes encargados de las diversas actividades (ludoteca, taller de naturaleza y juegos, zumba, taller de maquillaje y peluquería, etc.). La referente territorial se posicionó en la estructura de gestión con dos roles: por un lado, asumiendo la responsabilidad de garantizar, con el apoyo de su propia red, la asistencia alimentaria a los grupos familiares que asistían a la institución; por otro lado, se sumó a la estructura operativa del espacio en el rol de encargada de mantenimiento.

En otro orden, un convenio de obra vinculado con el mismo programa financió la ampliación del edificio de acuerdo con las necesidades (figura 3). El espacio se pensó esta vez como un centro socioeducativo. Para la finalización de la obra, el ministerio ejerció cierta presión para que el espacio se inaugurara a finales de julio de 2019, previamente a las elecciones presidenciales (11 de agosto de 2019). Finalmente, la sede comenzó a funcionar el 10 de agosto de 2019, y en el acto de inauguración participaron vecinos y vecinas, sin presencia de las autoridades políticas.

Desde su inauguración, el dispositivo ha funcionado como un núcleo de participación familiar y ciudadana, ofrece actividades recreativas, artísticas, deportivas, informativas y, a través de las vinculaciones con otros organismos o instituciones, el acceso a servicios tales como educación y salud.
7. https://www.gba.gob.ar/ desarrollosocial/asistencia/ casas_de_encuentro. Acceso: 27 octubre de 2019. 
Figura 3. Ampliación Casa de Encuentro Comunitario

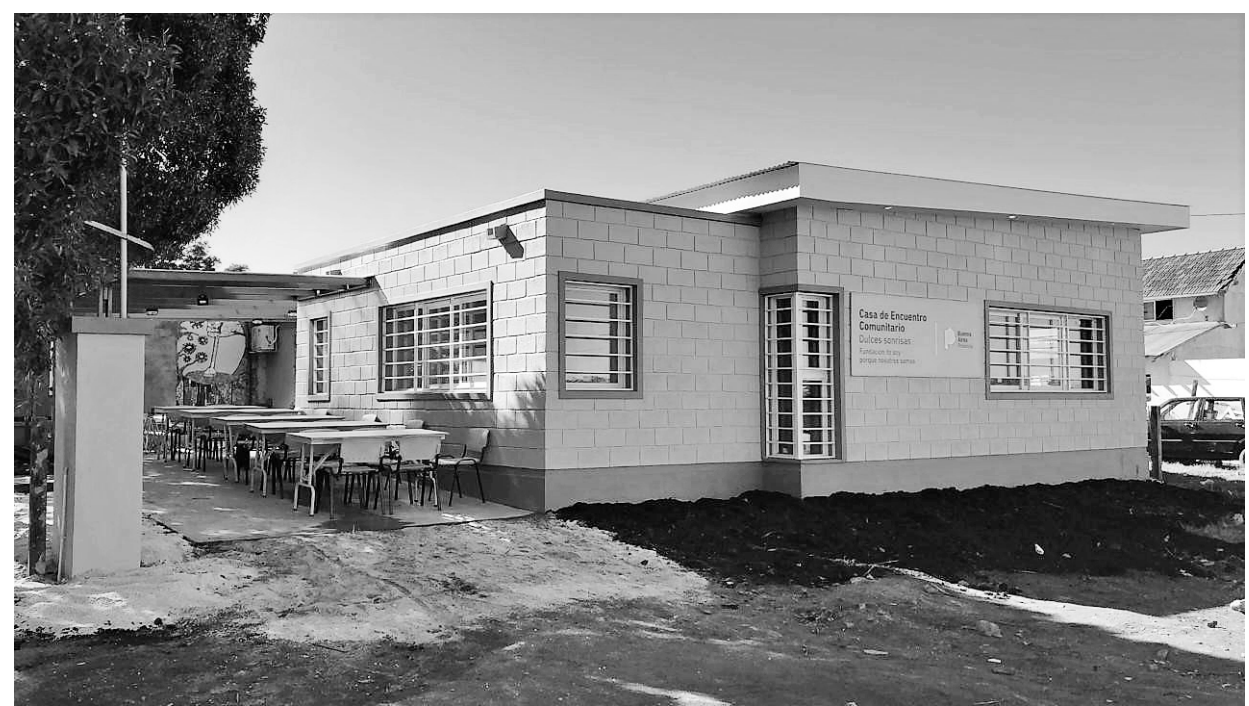

El programa CEC posee un acuerdo de comodato entre la ONG y la poseedora del terreno, lo que posibilita una perspectiva de funcionamiento de seis años, aunque en la práctica ha sido necesaria una renovación anual del subsidio para sostener al equipo técnico-profesional y los talleristas.

\section{Organismo Provincial de Integración Social y Urbana}

En mayo de 2019, ante un auditorio compuesto por empresarios y miembros de cámaras de empresas, se anunció el desembarco del Organismo Provincial de Integración Social y Urbana (OPISU) a la ciudad de Mar del Plata. A cargo directamente de la Secretaría de Gobierno de la Provincia de Buenos Aires, el organismo fue creado en 2018 para dar respuestas a las problemáticas socio-habitacionales en barrios con "asentamientos informales" en diecisiete localidades de la provincia. En Mar del Plata se instalaron dos dispositivos que cubrían tres barrios con alto grado de vulnerabilidad social, habitacional y urbana. El objetivo de estos dispositivos era generar mesas participativas con los vecinos para la detección 
de problemáticas y realizar tareas de atención temprana dedicadas a la limpieza y mantenimiento de espacios públicos y calles. Según el testimonio de una funcionaria, las actividades que llevarían adelante incluían un mapeo socio-problemático en forma colaborativa con los vecinos para elaborar un "máster plan” de "urbanización” del barrio.

La llegada del organismo en el barrio se materializó en un container donde funcionó su oficina por cinco meses (figura 4). La estructura de gestión estaba constituida por tres técnicos - dos arquitectos y una trabajadora social-y una voluntaria que de lunes a viernes recibían a los vecinos y se encargaban de organizar las tareas de las cuadrillas y relevar el estado de situación del barrio. Las actividades comenzaron a desarrollarse en el mes de junio de 2019, con la conformación de dos equipos de trabajo para abordar las tareas tempranas. Se destacan tres de las "tareas tempranas" llevadas adelante por el organismo:

1. El mantenimiento de espacios públicos, pintura de juegos de plaza y cordón, limpieza de cordón cuneta y de microbasurales, a partir de la contratación de una cooperativa que alquiló su figura jurídica a la provincia y asoció a veintiséis vecinos y vecinas del barrio.

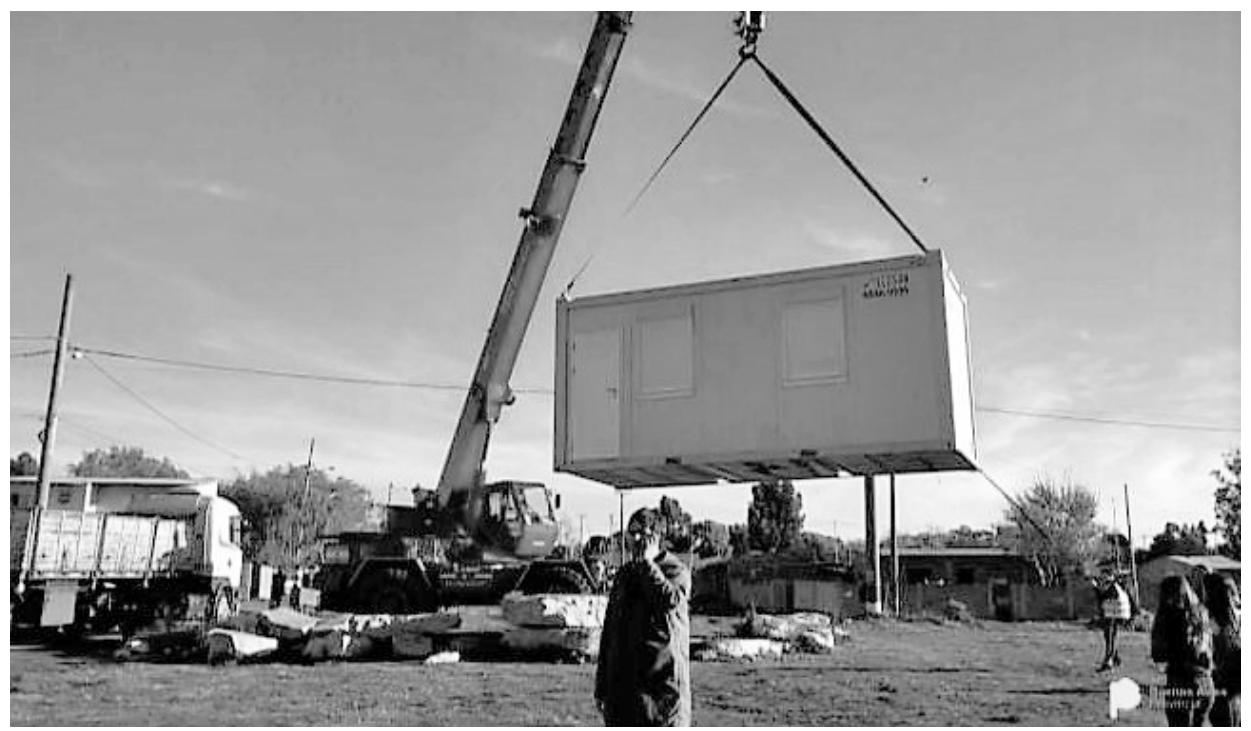

8. La estrategia de implementar el OPISU en Mar del Plata se anunció a principios de 2019 en el Museo de Arte Contemporáneo MAR, sin presencia de la prensa, que en sus declaraciones anunció un plan que tenía como fin urbanizar y mejorar la infraestructura de tres barrios vulnerables de la ciudad: Nuevo Golf, Autódromo y Belgrano. Ver: https://cypnoticias.com. ar/2019/04/11/urbanizarantres-villas-en-mar-del-plata/. Acceso: 12 de noviembre de 2019.

Figura 4. Desembarco de OPISU en Nuevo Golf 
9. En: https://www.argentina. gob.ar/sites/default/ files/planes_programas sociales_y_prestaciones_de

la_seguridad_social_0_0. pdf. Acceso: 27 de octubre de 2019.
2. La contratación de dos camiones de desagote para vaciar los pozos de los hogares del barrio.

3. El relevamiento y entrega de 33 techos de chapa y tirantes a familias en situación habitacional crítica.

A tres meses de haber comenzado, el organismo concentró a un numeroso grupo de vecinos y vecinas para exponer la propuesta en una reunión organizada en el edificio de la CEC. Presentaron los resultados obtenidos de las tareas tempranas y la elaboración de un ambicioso proyecto de urbanización. El plan de urbanización presentado como master plan incluía el mejoramiento y extensión de servicios, el proyecto de tendido de red cloacal, la ampliación de la red de agua, el tendido y alumbrado público, el mejoramiento del estado de calles y espacios públicos y la incorporación de equipamiento comunitario y sanitario. La modalidad de la exposición fue a través de una presentación en la cual mostraron “¿qué encontramos?, ¿qué queremos?, ¿qué hicimos?, ¿qué falta?”. Es importante destacar que varias de las iniciativas planteadas en la exposición como logros propios expuestos ya habían sido abordadas por la AV. Sin embargo, en su informe de urbanización integral el OPISU desconoció las gestiones previas.

Los contratos del personal técnico tuvieron una duración de seis meses, con una finalización planificada para el 30 de diciembre de 2019, aunque el equipo cesó sus actividades en territorio a mediados de noviembre de 2019, una vez resueltas las elecciones ejecutivas.

\section{Plan Nacional de Protección Social}

Desde el año 2012 el grupo de investigación se ha vinculado con la Asociación Vecinal para trabajar en forma conjunta en procesos de gestión a nivel local y en el acompañamiento de procesos habitacionales (asistencia para la ampliación y mejoramiento de la sede vecinal, articulación y co-construcción de un espacio comunitario para las actividades recreativas, conexiones eléctricas seguras, etc.) (figura 5, página siguiente).

En 2017, el Ministerio de Salud y Desarrollo Social de la Nación impulsó el Plan Nacional de Protección Social (PNPS), con la finalidad de aportar herramientas que posibiliten el desarrollo personal y grupal de hogares en estado de extrema vulnerabilidad ${ }^{9}$. Se planteaban como ámbito de aplicación los espacios donde funcionaran Casas de Encuentro Comunitario. A comienzos de 2019, el ministerio decidió implementar este plan en Mar del Plata 
Políticas públicas y participación ciudadana: análisis de tres programas estatales implementados en un barrio popular de Mar del Plata (2018-20२०)

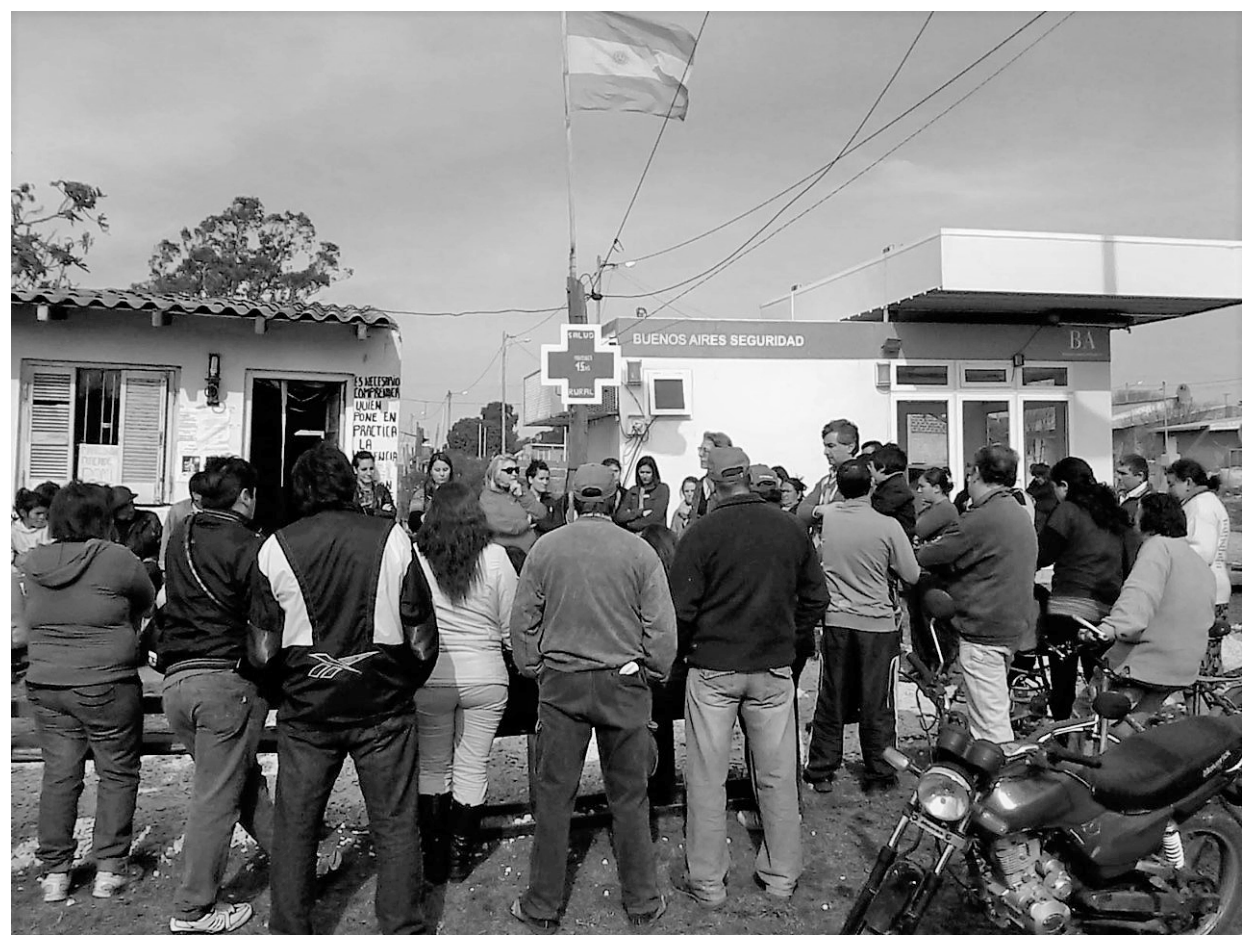

Figura 5. Reunión

vecinal en sede de $A V$

junto con la ONG que coordinaba el funcionamiento de la CEC en Nuevo Golf. La estrategia de la ONG y del grupo de investigación fue ejecutar el plan vinculado con las actividades preexistentes de la AV.

La decisión de ejecutar el PNPS en conjunto con la AV fue estratégica en tres órdenes:

1) Los actores del grupo de investigación y la ONG reconocían la trayectoria de lucha territorial del referente de la AV en el mejoramiento de la calidad de vida urbana, habitacional y de acceso a servicios de la población del barrio. 
2) Los objetivos propuestos por el plan ya eran llevados adelante a través de gestiones vecinales y estrategias colectivas (programas de salud rural, reclamos barriales, actividades recreativas y culturales).

3) El programa CEC se había constituido como dispositivo relevante en el barrio y había una necesidad creciente de equilibrar las disputas de poder emergentes entre ambas instituciones.

Si bien el PNPS no exigía la existencia de una sede fija, la AV se configuró como espacio de reunión para sus coordinadores e integrantes, y junto con la CEC funcionaron como sedes para la realización de talleres que comenzaron a partir de noviembre de 2019. El PNPS en sus objetivos principales planteaba materializar tres ejes de trabajo. Por un lado, el "acompañamiento familiar" a través de un seguimiento semanal enunciado por el plan como "un proceso de promoción social que atiende en forma directa a los hogares en situación de extrema vulnerabilidad social, con el objetivo de movilizar sus capacidades, generar oportunidades y promover su autonomía”. En la práctica, estas visitas servían para promover el acceso a la seguridad social a través de gestiones de DNI y programas sociales. Por otro lado, el "desarrollo comunitario", un eje para "fortalecer las capacidades de la comunidad para abordar problemáticas de forma conjunta y planificada, promover la participación, los espacios de diálogo, proyectos colectivos, entre otros aspectos". Se implementó a partir de cursos de formación en oficios de tres meses. Por último, el eje "recursos para la promoción social”, materializado en acciones para promover la seguridad alimentaria en los hogares de extrema vulnerabilidad social y en la transferencia de recursos a través de la tarjeta alimentaria. Este último eje, presente en todo el territorio nacional del plan, fue descartado en la provincia de Buenos Aires, con el argumento (extraoficial) de superposición con la estructura de la CEC y falta de fondos suficientes para una cobertura en la provincia.

La estructura organizativa prediseñada estaba compuesta por un coordinador general, dos coordinadores de área, ocho acompañantes familiares, personal administrativo y talleristas. Los acompañantes familiares fueron seleccionados por los referentes de la AV, a la vez coordinadores del plan, estrategia promovida por la ONG para aumentar los grados de libertad de la AV al elegir “compañeros” con afinidad y trayectoria dentro del espacio.

La implementación se planteó con un período de ejecución de doce meses, desde julio de 2019 a julio de 2020. La etapa de acompañamiento se dio desde el inicio del plan, y la etapa de desarrollo comunitario comenzó luego del período de diagnóstico de tres meses 
cubriendo con talleres rotativos los nueve meses restantes. En cuanto a las herramientas, desde el ministerio se proveyó a coordinadores y acompañantes de un documento en PDF llamado "recursero", con alrededor de 180 números de teléfonos y correos de oficinas o dependencias, para ser utilizado en distintas problemáticas que pudieran surgir en el proceso de acompañamiento de las familias del barrio.

La primera acción consistió en un relevamiento a 210 familias en estado de vulnerabilidad socio-habitacional ${ }^{10}$ a través de una aplicación móvil que tomó algunas horas descargar, puesto que demostraba dificultades en versiones del principal sistema operativo de uso masivo en Argentina. Los acompañantes debían contar con un dispositivo propio, cargar allí los datos relevados y una vez finalizada la encuesta acercarse a un punto con señal de internet para subir la información a un servidor. Parece difícil en un barrio con una red de cobertura de datos deficiente pensar en la modernización planteada por los aplicativos móviles, pero, aun así, fue posible la carga de datos desde la sede de la AV. Luego de un "filtro" realizado por el ministerio, se comenzó a trabajar con las 160 familias que estaban en condiciones más desfavorables ${ }^{11}$. Por otro lado, en función del acuerdo con la entidad vecinal, y tomando como referencia los ejes de trabajo del plan, la ONG y el grupo de investigación propusieron los talleres destinados al aprendizaje de oficios relacionados con la construcción y la promoción de la salud integral que se desarrollaron en los próximos meses.

Para organizar la información expuesta y aproximar al entendimiento de cómo se adapta al territorio cada política pública desarrollada, se presenta un cuadro comparativo sobre las dimensiones de análisis (figura 6, página siguiente).

\subsection{Articulaciones en la implementación de los programas}

Retomando la discusión sobre qué es o no es política pública, lo que es visto usualmente desde los organismos estatales como una política de inclusión de asentamientos informales aquí se deconstruye en complejas redes de interacciones barriales e interinstitucionales que se vinculan con prácticas de resistencia y confrontación. En estas redes de actores organizadas alrededor de cada programa, ocurre un proceso de negociación en el cual se distribuyen los roles en tareas y responsabilidades. Se crea aquí una difusa división social del trabajo en la cual se imbrican diferentes formas jurídicas organizativas (organizaciones vecinales, ONG, Asociaciones civiles, Cooperativas) —además del Estado-, en pos de poner en práctica subsidios, distribuir trabajo y solucionar los problemas existentes.
10. De las 1000 familias que viven en Nuevo Golf, las 210 relevadas fueron seleccionadas por el coordinador del plan, en este caso, el vecinalista y su equipo.

11. El mecanismo de selección de las 160 familias merece un estudio aparte que no será abordado en el artículo, pero es necesario subrayar que el relevamiento de las 210 familias iniciales sufrió un proceso de "filtro" realizado por técnicos del ministerio a través de un programa que dejó afuera a familias que estaban en una situación de vulnerabilidad mayor comparadas con varias familias que sí fueron "elegidas" dentro de las 160. 
Figura 6

\begin{tabular}{|c|c|c|c|}
\hline \multirow{2}{*}{$\begin{array}{l}\text { Dimensiones } \\
\text { de análisis }\end{array}$} & \multirow[b]{2}{*}{ CEC } & \multirow[b]{2}{*}{ OPISU } & \multirow[b]{2}{*}{ PNPS } \\
\hline & & & \\
\hline $\begin{array}{l}\text { Trayectoria } \\
\text { socio-técnica }\end{array}$ & $\begin{array}{l}\text { Desde } 2013 \text { como comedor } \\
\text { barrial. Presencia de ONG en } \\
\text { el mejoramiento habitacional } \\
\text { del espacio comunitario }\end{array}$ & Sin trayectoria & $\begin{array}{l}\text { Desde } 2010 \text { la AV desarrolla } \\
\text { actividades abordando distintos } \\
\text { aspectos que hacen al desarrollo } \\
\text { local del barrio. Presencia de la } \\
\text { ONG en el acompañamiento }\end{array}$ \\
\hline $\begin{array}{l}\text { Alcance } \\
\text { del trabajo }\end{array}$ & $\begin{array}{l}\text { Desarrollo social, asistencia } \\
\text { alimentaria y actividades } \\
\text { recreativas. }\end{array}$ & Mejoramiento Urbano & Desarrollo social y territorial \\
\hline $\begin{array}{l}\text { Modalidad de } \\
\text { participación } \\
\text { (rol- forma } \\
\text { jurídica) }\end{array}$ & $\begin{array}{l}\text { Comedor barrial - La referente } \\
\text { del espacio comunitario pasa } \\
\text { a formar parte de equipo } \\
\text { contratado por ONG }\end{array}$ & $\begin{array}{l}\text { Armado de cuadrillas de } \\
\text { trabajo y cooperativas } \\
\text { contratadas. Sin referente } \\
\text { territorial }\end{array}$ & $\begin{array}{l}\text { AV- Los referentes de la AV y } \\
\text { espacio político se convierten en } \\
\text { coordinadores del plan }\end{array}$ \\
\hline $\begin{array}{l}\text { Momento de } \\
\text { implementación }\end{array}$ & $\begin{array}{l}\text { Julio de } 2018 \text { a julio de } 2024 \\
\text { Obra de ampliación: } \\
\text { diciembre de } 2018 \text { - agosto de } \\
2019\end{array}$ & $\begin{array}{l}\text { Julio de } 2019 \text { a } \\
\text { diciembre de } 2019\end{array}$ & Julio de 2019 a julio de 2020 \\
\hline $\begin{array}{l}\text { Período de } \\
\text { ejecución }\end{array}$ & $\begin{array}{l}\text { Horizonte a seis años con } \\
\text { período de renovación anual }\end{array}$ & $\begin{array}{l}\text { La labor del equipo técnico } \\
\text { se vio se vio interrumpida en } \\
\text { noviembre } 2019 . \text { Las cuadrillas } \\
\text { completaron el ciclo. }\end{array}$ & $\begin{array}{l}\text { Período de } 3 \text { meses de } \\
\text { relevamiento y } 9 \text { meses de } \\
\text { acompañamiento familiar y } \\
\text { desarrollo comunitario }\end{array}$ \\
\hline $\begin{array}{l}\text { Recursos } \\
\text { materiales y } \\
\text { humanos }\end{array}$ & $\begin{array}{l}\text { *Subsidio de obra para } \\
\text { ampliar } \\
\text { *Talleres } \\
\text { *Subsidio alimentario } \\
\text { (alimentos secos y leche) } \\
\text { *Equipo profesional }\end{array}$ & $\begin{array}{l}\text { *Tareas tempranas (pintura } \\
\text { de aceras, limpieza de micro } \\
\text { basurales, mejoramiento de } \\
\text { calles manualmente, limpieza } \\
\text { de plazas y reparación juegos } \\
\text { y mobiliario) } \\
\text { *Plan de desarrollo integral } \\
\text { (Máster plan) }\end{array}$ & $\begin{array}{l}\text { *APP relevamiento } \\
\text { *Tarjeta alimentar } \\
\text { *Recursero del estado } \\
\text { *Talleres } \\
\text { *Acompañamiento familiar }\end{array}$ \\
\hline
\end{tabular}

Fuente: elaboración propia 
El sociograma (figura 7) pretende aportar como dato de interés la articulación entre los actores sociales. Estos son representados por círculos de diferentes tamaños que simbolizan el grado de participación dentro de la red de gestión. Las líneas que unen los distintos actores indican los tipos de articulaciones, que pueden ser continuas, ocasionales o disfuncionales. Además, en cada articulación se especifica la naturaleza del vínculo y los flujos.

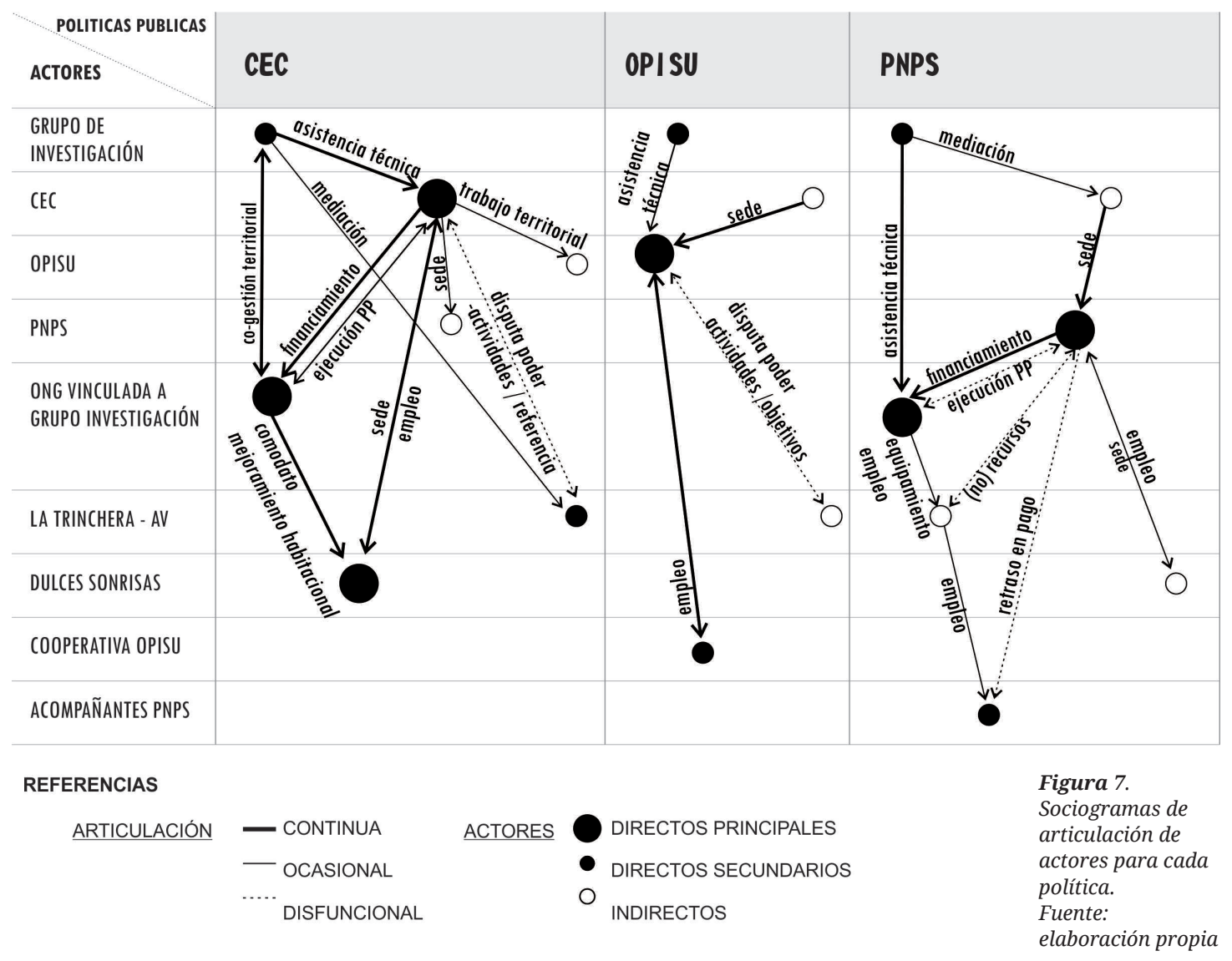


12. El anuncio de la creación de OPISU, en los medios de comunicación, indicaba al ente como encargado de

llevar a cabo las gestiones para la llegada de los servicios básicos (agua potable, cloacas, red eléctrica, gas natural), apertura de calles y la urbanización de los barrios.

https://www.lacapitalmdp. $\mathrm{com} /$ proyectan-la-

urbanizacion-y-el-desarrollode-tres-barrios-de-mar-del-

plata
Se identifican articulaciones disfuncionales que ayudan a entender los problemas de interacción de las políticas en los territorios que se analizan a continuación.

\section{Casa de Encuentro Comunitario: la integración de lo preexistente}

En la experiencia CEC, la política pública se incorporó al proceso de un comedor y espacio de usos múltiples existente, con una estructura que permitió financiar actividades y potenciar el alcance de los objetivos planteados. La estructura organizativa facilitó el trabajo en conjunto con escuelas, centros de atención primaria de la salud, iglesias, grupos universitarios, diversas ONG y organismos estatales. La incorporación de profesionales (psicólogos, psicopedagogos, trabajadores sociales, docentes) y la articulación fluida con herramientas disponibles de la provincia en materia de desarrollo social posicionaron este espacio como referencia para gran parte del barrio.

Sin embargo, se produjeron disfuncionalidades con la AV, que impulsaba prácticas similares a menor escala y con recursos propios. El referente de la AV entendía que las actividades se yuxtaponían y no se complementaban; además consideraba que existía poca coordinación entre agendas (entrevista semiestructurada, 19 de octubre de 2019).

A pesar de los intentos de consensuar agendas y compartir carteleras de ambos espacios, tanto la AV como la CEC funcionan sin coordinar actividades entre ellas. En este punto, la tensión generada es mediada por la ONG y el grupo de investigación a partir de la propuesta de implementar el PNPS en la AV, como una estrategia de equilibrar las disputas de poder entre ambas instituciones.

\section{Organismo Provincial de Integración Social y Urbana:}

\section{de la "integración urbana" a las "tareas tempranas"}

La mayor desarticulación en la implementación de este programa se produjo en relación con la AV al no reconocer y potenciar el trabajo de esta en materia de mejoramiento urbano. El organismo cuyo nombre representaba el imaginario del acceso a la urbanización formal disputaba gestiones barriales a la escala de una asociación vecinal ${ }^{12}$. En una mesa de discusión realizada entre el referente de la AV, funcionarias y funcionarios de OPISU y participantes del grupo de investigación, previamente a la instalación de este dispositivo en el barrio, se rescata un diálogo relevante acerca del concepto de urbanización. Una funcionaria se limitaba a hacer hincapié en la futura realización de "tareas 
tempranas" con cuadrillas de limpieza y mantenimiento, mientras que el referente de la AV discutía el alcance de las "tareas tempranas". El referente entendía que la urbanización debería incluir acciones para resolver problemas como el acceso a salud, educación e infraestructura de servicios:

Desde este espacio organizado se reclama la reorganización de la agenda de la política, para que alcance ejes no desarrollados, para evitar esfuerzos innecesarios en gestiones que la comunidad organizada ya logró... No tiene sentido poner un contenedor en el barrio, para que averigüen qué problemas tienen los vecinos, ya que los problemas que tenemos están a la vista, los hemos visibilizado de todas las formas posibles, al punto de que su propio equipo caminó nuestras calles. Realmente solo se necesita de la decisión política para que estos problemas sean finalmente resueltos (conversaciones entre referente de la AV, funcionarios OPISU y grupo de investigación).

Por mencionar otra de las disfuncionalidades, en el master plan de OPISU se proyectaba el tendido de la red cloacal, cuando, en realidad, el análisis de factibilidad del proyecto y la cotización de obra ya habían sido realizados por la AV con anterioridad ${ }^{13}$.

Sobre las necesidades del barrio, el grupo de investigación refuerza la idea de urbanización referida a la infraestructura como fundamental. También remarca la estrategia de vinculación necesaria entre la provincia con "los organismos locales con los que el centro vecinal ya ha establecido gestiones y avances. Ese es el punto innovativo para hacer una gestión efectiva” (conversaciones entre referente de la AV, funcionarios OPISU e investigadores).

Desde el punto de vista técnico, los objetivos planteados eran irrealizables en un plazo tan reducido. Las tareas tempranas de mejoramiento de calles y juegos de plaza fueron la antesala para exponer ante los vecinos el master plan del barrio. Para su desarrollo el presupuesto estimado era de 211 millones de pesos, y su realización, según lo expuesto por el equipo técnico en la presentación, "sería responsabilidad de los que vienen que lo quieran financiar" (funcionaria OPISU en conversaciones entre el presidente de la Asociación Vecinal Barrio Nuevo Golf, funcionarios OPISU y el grupo de investigación, Mar del Plata, 14 de mayo de 2019). El oportunismo político tardío para sumar poder al régimen vigente, materializado en la promesa de desarrollo integral a un territorio, tuvo un abrupto fin en un muy corto plazo.
13. En una reunión entre el referente de la $A V$ y las autoridades de OSSE (Obras Sanitarias Mar del PlataBatán) del día 6 de abril de 2018, se firmó un acta donde la empresa se comprometió a elaborar las opciones técnicas del proyecto de redes cloacales. OSSE realizó el proyecto y el cómputo por el tendido de cañería con la observación de que en ese momento no se contaba con fondos para su ejecución. 


\section{Plan Nacional de Protección Social: el discurso político vs. el saber hacer local}

En la experiencia PNPS, las disfuncionalidades estuvieron asociadas con problemas inherentes a la burocracia del programa que generaron tensiones entre representantes del plan y de la AV, los responsables de la ONG y los técnicos del programa (funcionarias y funcionarios). Ante la necesidad de modificar los talleres planificados antes de conocer el perfil de las y los participantes, el equipo de gestión tuvo que enfrentarse con un mecanismo sumamente tedioso. Este hecho, sumado a un retraso importante en la transferencia de fondos -que implicó meses de trabajo sin que los acompañantes cobraran—, generó desgaste en la implementación, que exigía una "transparencia" disfuncional a las necesidades emergentes.

Por otro lado, el seguimiento de las familias que recibían "acompañamiento familiar" fue difícil de concretar, debido a que el programa carecía de herramientas necesarias o eran insuficientes por dos motivos: 1) el "recursero" tenía una lista interminable de teléfonos que correspondían a la administración central (con sede en CABA), que en gran medida no daba respuestas, por lo que no fue considerada una herramienta de apoyo eficiente por las acompañantes y 2) haber descartado el eje "recursos para la promoción social", materializado en una tarjeta alimentaria, dificultaba la motivación para dejarse acompañar. En un encuentro con acompañantes familiares, integrantes del grupo de investigación y un funcionario del ministerio, se rescata el siguiente diálogo:

Es muy difícil querer acompañar a una familia si no hay nada a cambio para ofrecerle, si está en una situación de vulnerabilidad y no tiene para comer... entonces después de una o dos visitas, deja de recibir al acompañante y pierde interés en participar en el programa (...) necesitamos alguna especie de paraguas para darle un impulso a las familias, una contención (...) el recursero es un instrumento que te dice cómo tenés que hacer los trámites y te da un teléfono, pero no te da soluciones. Las cosas que sugiere ya sabemos por nuestra propia experiencia que no funcionan, Desarrollo Social en Mar del Plata está tomado hace meses (acompañante familiar, Mar del Plata, 25 de septiembre de 2019).

Esta manifestación de las disfuncionalidades en la implementación tuvo la siguiente respuesta del funcionario:

Ustedes ya sabían que la tarjeta no venía y el plan nunca les prometió una tarjeta. Si no creen en la promoción del hogar sin apoyo económico, eso el plan nunca se los va a dar. Si ahora piensan que estamos cambiando la bocha, damos de baja el programa y listo...Yo para resolver estos pro- 
blemas que me plantean apelo a su creatividad [cursivas añadidas], es así... Es disruptivo lo que estamos planteando desde este programa: promoción social sin asistencia material (funcionario PNPS, Mar del Plata, 25 de septiembre de 2019).

En la respuesta del funcionario se contrapone su visión del asistencialismo con los propios ejes del plan, que incluía en su diseño una línea de asistencia alimentaria. El diálogo rescatado refleja la incomprensión de las problemáticas barriales, sobre las cuales la AV y las acompañantes familiares tenían un profundo conocimiento. Este saber local se convirtió en la herramienta más eficiente que el programa tuvo.

\section{Reflexiones finales}

Se retoma aquí la pregunta planteada en la introducción de este trabajo: ¿cómo pensar políticas de integración socio-urbana vinculada con territorialidades locales? El nexo espacial y temporal común de estos programas permitió reflexionar sobre la interrelación de los actores intervinientes e identificar aspectos funcionales y disfuncionales que contribuyeron al desarrollo barrial de las políticas. De acuerdo con Peyloubet (2014), se entiende el saber de sentido común como productor de la resolución de los múltiples problemas que los sujetos enfrentan a diario en su vida cotidiana. Este saber toma relevancia en la implementación de políticas sociales en las cuales los actores locales que ejercen roles de representación barrial tienen un profundo conocimiento no solo del territorio entendido como espacio geográfico, sino de las redes e instituciones que operan sobre ese espacio y, también, sobre el funcionamiento de las dependencias locales y municipales.

Entre los actores locales se reconocen diferencias y matices, de acuerdo con su legitimidad y capacidad de gestión. Un primer paso para pensar políticas de integración socio-urbanas con mayor anclaje territorial debería ser la identificación y localización de las capacidades locales, ya sea en una asociación vecinal, un comedor, una institución estatal u organización social, entre otras posibilidades. Sin embargo, el objetivo no es identificar actores con capacidades "necesarias", noción que operaría como barrera de entrada. Por el contrario, el objetivo sería indagar potenciales capacidades y sus articulaciones. De estas capacidades dependerá la efectividad de utilización de los recursos disponibles, que circularán fortaleciendo redes y caminos previamente construidos. Así también, se disminuirá el riesgo de que una política sea funcional a la reproducción de otros intereses ajenos al territorio. 
En cuanto a las vinculaciones, estas no pueden ser vistas como una caja negra que no explicite la naturaleza de los vínculos entre los actores. Es por eso que en el presente análisis se hace hincapié en los aspectos funcionales y disfuncionales, en los conflictos de intereses y de saberes, donde se conjugan múltiples tensiones que deben ser analizadas.

Entonces, ¿qué sentido tienen estas reflexiones sobre los resultados presentados como insumo para una política pública? Como aproximación y desafío, es necesario comprender e interpelar el rol del Estado en la aplicación de planes y programas de gestión que discursivamente coinciden con la denominada gestión alternativa, en el sentido de que buscan incorporar una perspectiva interactoral, multisectorial y participativa para el abordaje de los problemas sociales existentes. Por su lógica de acción, con sus instituciones y materialidades, observamos que tanto el Estado como institución como sus funcionarios/as que actúan como portavoces de una determinada gestión buscan vincularse y generar autonomía y empoderamiento de las organizaciones. No obstante, a la vez presionan y obstruyen con procesos burocráticos y políticos desterritorializados funcionales a la reproducción de sus propias estructuras.

En los programas presentados encontramos desde grados de libertad amplios para los actores locales, con posibilidades de diseñar los equipos de trabajo, talleres y metas propias para alcanzar objetivos y potenciar redes existentes, hasta el uso de estos programas para diseñar un master plan de urbanización, ajeno a toda posibilidad real, que termina siendo funcional a un interés de captación de clientela política. El Estado tiene un rol importante en estos procesos multiactorales. Su presencia incide en las relaciones sociales previas favoreciendo o con procesos disruptivos. Es decir, así como el desarrollo de estos planes y programas puede contribuir a transformar las condiciones de vida de la población en situaciones de pobreza, también puede contribuir a la reproducción de esas condiciones. El desafío aparece, como la necesidad de construir nuevos saberes sobre los actores, sus interacciones y las formas de distribución de poder.

Las dimensiones de análisis planteadas y sus articulaciones instalan a los territorios y los actores locales en el centro de la escena para definir sus estrategias de desarrollo. Finalmente, sobre estas experiencias se intentó alguna contribución, tanto a teoría en el campo de la urbanización popular como el de políticas públicas, a partir del análisis de las capacidades organizativas locales y sus vinculaciones. 


\section{Referencias bibliográficas}

Cacopardo, F.; Rotondaro, R.; Blanco Pepi, M.; Cacopardo, G.; Freire, P.; Ispizúa, J.; Melián, I. \& Mitidieri, A. (2018). Tecnologías sociales en territorios urbanos pobres. Barrio Nuevo Golf, Mar del Plata, Argentina (2010-2018). Redes. 24(47), 227-262. https://revistaredes.unq.edu.ar/index.php/redes/ article/view/37

Cravino, M. C. \& Varela, O. (2010). Mil nombres para mil barrios: asentamientos y villas como categorías de análisis e intervención. En M. C. Cravino (Ed.), Los mil barrios (in)formales: Aportes para la construcción de un observatorio del hábitat popular del Área Metropolitana de Buenos Aires (pp. 45-64). Los Polvorines, Argentina: Universidad Nacional de General Sarmiento. https://periferiaactiva.files.wordpress.com/2015/06/los-mil-barrios-cravino-final.pdf

Gargantini, D.; D’Amico, D. \& Martiarena, M. (2018). El gobierno del suelo urbano: representaciones y estrategias de articulación-acción de los actores estatales. Revista Territorios (38), 119-136. https://doi.org/10.12804/revistas.urosario.edu.co/territorios/a.5285

Mitidieri, L. A.; Ispizúa, J. \& Blanco Pepi, M. (2019). Estrategias de gestión y redes de interacción en la urbanización popular. Barrio Nuevo Golf, Mar del Plata, Argentina. Segundas Jornadas de Sociología UNMDP. https://fh.mdp.edu. ar/encuentros/index.php/jsoc/jsoc2019/paper/view/5608.

Oszlak, Oscar \& O'Donnell, Guillermo (1995). Estado y políticas estatales en América Latina: hacia una estrategia de investigación. Redes, 2(4), 99-128. https://www. redalyc.org/articulo.oa?id=907/90711285004.

Oszlak, O. (2009). Implementación participativa de políticas públicas: aportes a la construcción de un marco analítico. En Belmonte, Alejandro (et al.). Construyendo confianza. Hacia un nuevo vínculo entre Estado y Sociedad Civil, Volumen II (pp. 9-47). Fundación CIPPEC. https://www.cippec.org/wp-content/ uploads/2017/03/1753.pdf

Pelli, V. (2007). Habitar, Participar, Pertenecer. Acceder a la vivienda-incluirse en la sociedad. Nobuko.

Peyloubet, P. (2012). Hacia una Base Cognitiva Democrática - Debate, Construcción y Operacionalización del Conocimiento Colectivo en el Campo del Hábitat. En 
Peyloubet, P. (Ed.), Co-construcción interactoral del conocimiento (pp.153170). Nobuko. https://coconstrucciondelconocimiento.files.wordpress. com/2015/06/const_int_conoc.pdf

Peyloubet, P. (2014). Del rango epistémico al saber de sentido común. Revista de Antropología Experimental, 0(14), 55-81.

Peyloubet, P. (2017). Animarse a habitar. Diseño.

Pirez, P. (1995). Actores sociales y gestión de la ciudad. Ciudades, 28, 1-12. RNIU.

Pirez, P. (2015). Servicios urbanos y urbanización popular: mercantilización y desmercantilización. En Bolivar, T.; Rodríguez, M. \& Erazo, J. M. (Ed.), Ciudades en construcción permanente ¿Destino de casas para todos? (pp. 55-79). CLACSO.

Subirats Humet, J.; Knoepfel, P.; Larrue, C. \& Varone, F. (2008). Análisis y gestión de políticas públicas. Ariel.

Thomas, H.; Fressoli, M. \& Santos, G. (2012). Tecnología, desarrollo y democracia: nueve estudios sobre dinámicas socio-técnicas de exclusión/inclusión social. Ministerio de Ciencia, Tecnología e Innovación Productiva e Instituto de Estudios sobre la Ciencia y la Tecnología, Universidad Nacional de Quilmes (IESCT-UNQ). 